\title{
ESTUDIO TRANSVERSAL PILOTO SOBRE EL PERFIL FUNCIONAL DEL PACIENTE EN CUIDADOS PALIATIVOS. ACTIVIDAD Y DESEMPEÑO OCUPACIONAL
}

\author{
Laura Carrasco-Tanarro, MSc ${ }^{1,2}$; María Gracia Carpena-Niño, $\mathrm{PhD}^{1}$; Bárbara Mendiburu-Aletti, PT³; \\ Miguel Gómez-Martínez, $\mathrm{PhD}^{1,4}$. \\ 1. Occupational Thinks Research Group. Instituto de Neurociencias y Ciencias del Movimiento. Centro Superior de Estudios Universitarios La Salle. \\ Universidad Autónoma de Madrid. Madrid. España. \\ 2. Centro ECOPLAR Aravaca. Madrid. España. \\ 3. Hospital Centro de Cuidados Laguna. Madrid. España. \\ 4. Instituto de Rehabilitación Funcional'y ciencias aplicadas al deporte. Centro Superior de Estudios Universitarios La Salle. Universidad Autónoma de \\ Madrid. Madrid. España.
}

\section{Correspondencia:}

María Gracia Carpena-Niño, OT, PhD. Facultad de Ciencias de la Salud. CSEU La Salle. Universidad Autónoma of Madrid. Calle La Salle, ${ }^{\circ} 10,28023$ Madrid, España Teléfono: (+34) 917401980 Fax: (+34) 913571730

E-Mail: mgcarpena@lasallecampus.es

\section{Conflicto de Intereses:}

Los autores del presente manuscrito declaran no presentar ningún conflicto de interés y además el presente estudio no ha sido presentado en ningún evento científico.

\section{Financiación:}

Los autores declaran no haber recibido financiación/compensación para el desarrollo de esta investigación.

Comité de Ética:

CSEULS-PI-053/2015.

DOI:

https://doi.org/10.37382/jomts.v1i1.8

\section{Recepción del Manuscrito:}

3-Diciembre-2019

Aceptación del Manuscrito:

18-Marzo-2019

Licensed under:

CC BY-NC-SA 4.0

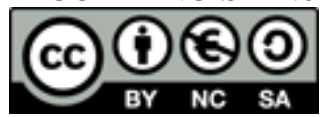

\section{RESUMEN}

Objetivo: Conocer el nivel de dependencia en actividades de la vida diaria del paciente que recibe cuidados paliativos, estudiando su relación con diferentes variables que pueden afectar en su estado funcional.

Diseño: Estudio piloto, observacional, transversal, de prevalencia, cuantitativo, prospectivo y analítico sobre la actividad y el desempeño ocupacional en pacientes de Cuidados Paliativos.

Marco: Se realizó en el marco hospitalario del centro de Laguna, Fundación VianorteLaguna.

Participantes: 34 pacientes cumplían los criterios de selección, pero no todos aceptaron participar. En la muestra se incluyeron 12 pacientes entre 49 y 89 años, diagnosticados de patología oncológica.

Intervenciones: Sin intervención.

Variables: Se estudiaron las siguientes variables cualitativas: edad, sexo, desempeño y satisfacción y cuantitativas: nivel de dependencia, calidad de vida, dolor, impacto de los problemas de salud y riesgo de deterioro de la integridad cutánea. Análisis estadístico mediante el programa SPSS 20.0.

Resultados: Las variables dolor, sueño, estado psicológico, continencia, úlceras por presión, movilidad y energía, se correlacionan de forma estadísticamente significativa $(\mathrm{p}<0,05)$ con el nivel de dependencia en las actividades de la vida diaria y su satisfacción.

Conclusión: Los pacientes que reciben cuidados paliativos presentan un perfil de dependencia severo en la realización de actividades de la vida diaria. Siendo el dolor, el sueño, el estado psicológico, las úlceras por presión, la movilidad y la energía, factores que limitan su desempeño ocupacional, principalmente en las actividades básicas de la vida diaria.

Palabras Clave: Calidad de vida, Cuidados paliativos, Rehabilitación, Terapia Ocupacional. 


\section{INTRODUCCIÓN}

En las últimas décadas ha aumentado la prevalencia de algunas enfermedades crónicas, junto al envejecimiento de la población. Los avances en medicina han aumentado la supervivencia y la Calidad de Vida (CV) de los pacientes, especialmente en los casos de las personas asistidas en unidades de cuidados paliativos (CP), donde se percibe con más claridad la cercanía de la muerte (Sociedad Española de Cuidados Paliativos, 2002).

La Organización Mundial de la Salud (OMS) define los CP como el "enfoque que mejora la $\mathrm{CV}$ de pacientes y familias que se enfrentan a los problemas asociados con enfermedades terminales, a través de la prevención y alivio del sufrimiento por medio de la identificación temprana, evaluación y tratamiento del dolor y otros problemas físicos, psicológicos y espirituales" (Mengual et al., 2005; Sociedad Española de Cuidados Paliativos, Ministerio de Sanidad Política Social e Igualdad, 2010).

Los enfermos oncológicos son los principales pacientes diana de los $\mathrm{CP}$, siendo el cáncer la principal causa de muerte en España (Sociedad Española de Cuidados Paliativos).Tienen alta prevalencia de dolor, fatiga, debilidad, disnea, depresión y ansiedad (Corregidor-Sánchez \& Ávila-Tato, 2010). Presentan limitaciones físicas como consecuencia de la enfermedad, haciéndose evidentes las repercusiones a nivel funcional y su impacto psicosocial debido a la proximidad de la muerte como problemas frecuentes presentan aislamiento y pérdida de independencia en las Actividades de la Vida Diaria (AVD) (Année, 2012). El objetivo terapéutico de los CP es proporcionar una atención integral dando mayor grado de bienestar a pacientes y familiares. La rehabilitación constituye un método no farmacológico para promover este bienestar (Gómez-Pavón \& Catalá, 2010), donde el paciente podría alcanzar el máximo grado físico, psicológico y social acorde a su situación clínica, sus limitaciones, sus deseos y sus expectativas (Corregidor-Sánchez \& Ávila-Tato, 2010), son aspectos diferenciales los que se articulan por los profesionales de los servicios de rehabilitación para ayudar a estas personas, como aspectos de asesoría, adaptación o modificación, y la terapia ocupacional, puede aportar aspectos que mejoren el bienestar de los sujetos.

La Federación Mundial de Terapeutas Ocupacionales define como principal objetivo de la Terapia Ocupacional (TO) la capacitación de las personas para participar en las AVD (WFOT, 2012).

Según un informe realizado por el Sistema de Información de Atención Primaria del Sistema Nacional de Salud en 2009, la TO no forma parte de las unidades de CP (Instituto de Información Sanitaria, 2010). La intervención de terapia ocupacional, sin embargo, permite observar la capacidad funcional real del paciente con los objetos y el entorno que dan una significación a sus actividades diarias, lo que favorece la motivación del paciente y los cuidadores y, por ende, la mejora de la calidad de vida. Respeta al máximo la historia y el estilo de vida del paciente, sus creencias, valores y gustos, fortaleciendo su sentido de pertenencia y control de su vida hasta que la enfermedad lo permita, lo que influye, de manera directa, en la calidad de vida del paciente (Astudillo Alarcón et al., 2008).

Para poder identificar las diferentes áreas de intervención es necesario identificar parámetros físicos como el dolor, psicológicos como la ansiedad y la depresión, cognitivos como el deterioro y, sobretodo, la percepción de eficacia de sus actividades cotidianas o de su calidad de vida que permita realizar una intervención más ajustada a sus necesidades específicas.

El objetivo principal del estudio es identificar los niveles de desempeño y dependencia de los pacientes en unidades de CP para las AVD.

\section{MÉTODOS}

\section{Diseño de estudio}

Se realizó un estudio observacional, transversal sobre actividad y desempeño ocupacional en pacientes 
de CP en el Hospital Centro de Cuidados Laguna, durante los meses de abril a junio de 2015 .

Es un centro promovido como entidad sin ánimo de lucro, la Fundación Vianorte-Laguna y, tiene un convenio con la Comunidad de Madrid, por lo que se reciben pacientes públicos y privados. El proyecto fue valorado positivamente por el Comité Ético de Investigación Clínica del Centro Superior de Estudios Universitarios de La Salle, CSEULS-PI-053/2015.

\section{Participantes}

Se incluyen pacientes ingresados en la unidad de $\mathrm{CP}$, que pueden comunicarse, con una puntuación en el Palliative Performance Scale (PPS) (Francis et al., 2008) de 40 o mayor a 40, que no presenten deterioro cognitivo (DC), y que no tengan Síndrome Confusional Agudo (SCA). Se excluyen pacientes pediátricos y pacientes atendidos a domicilio o en consultas externas.

Se seleccionaron los pacientes, y se les propuso participar en el estudio, tras la firma voluntaria del CI e realizó una única evaluación, entrevistando al paciente en su habitación y entregando a la familia un cuestionario. La valoración del paciente podía realizarse acompañado de familiares.

\section{Medidas de resultado}

Los instrumentos heteroaplicados a pacientes fueron:

\section{- Escala de CV (WHOQOL-BREF)}

Proporciona la opinión del paciente sobre su CV en su salud, el dolor o su satisfacción en diferentes áreas de la vida diaria proporcionando un perfil de CV percibida (The WHOQOL Grup, 1998).

\section{- Escala Visual Analógica (EVA)}

La escala EVA permite medir la intensidad del dolor percibido medido según una lineal en cuyos extremos se valora el "no dolor" y, en el extremo opuesto, "el peor dolor imaginable" (Corner, 1989).

\section{- Perfil de Salud del Nottingham (PSN)}

El PSN mide el sufrimiento físico, psicológico y social asociado a problemas médicos, sociales y emocionales y cómo influye ese sufrimiento en la vida diaria de los individuos (Alonso et al., 1994).

\section{- Medida Canadiense de Rendimiento Ocupacional (COPM)}

La COPM que evalúa el desempeño ocupacional y la satisfacción percibida por un individuo en las áreas de autocuidado, productividad y ocio (LindahlJacobsen et al., 2015).

El autoadministrado a familiares: Índice de Barthel (IB) es una medida genérica que valora el nivel de independencia del paciente respecto a la realización de algunas actividades básicas de la vida diaria, mide el grado de capacidad para llevar a cabo esas actividades (Barrero-Solís et al., 2005). A través de la historia clínica del paciente se recogieron los datos de la Escala Norton que valora el riesgo de deterioro de la integridad cutánea y de la presencia de factores de riesgo para el desarrollo de las úlceras por presión (UPP) (Instituto Nacional de Salud, 1996).

\section{Análisis estadístico}

El análisis estadístico se realizó mediante el programa IBM SPSS Statistics 20.0 (SPSS Inc., Chicago, IL, EE.UU.), con un análisis descriptivo y un análisis de correlaciones (mediante el Coeficiente de Spearman, considerando un nivel de significación $\mathrm{p} \leq 0.05$.

\section{RESULTADOS}

Teniendo en cuenta los criterios de inclusión y exclusión, fueron seleccionados 12 pacientes con patología oncológica, cuyas características demográficas se pueden ver en la Tabla 1.

Tabla 1. Características demográficas

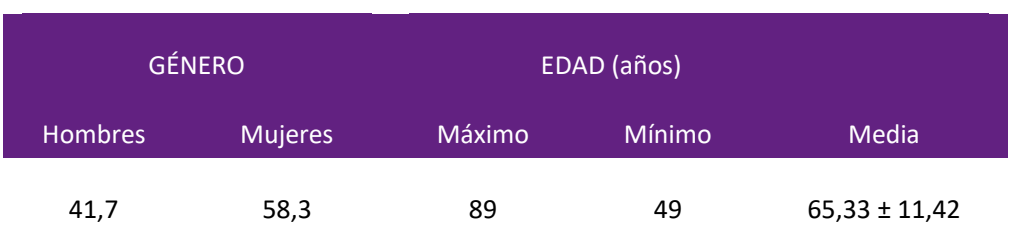




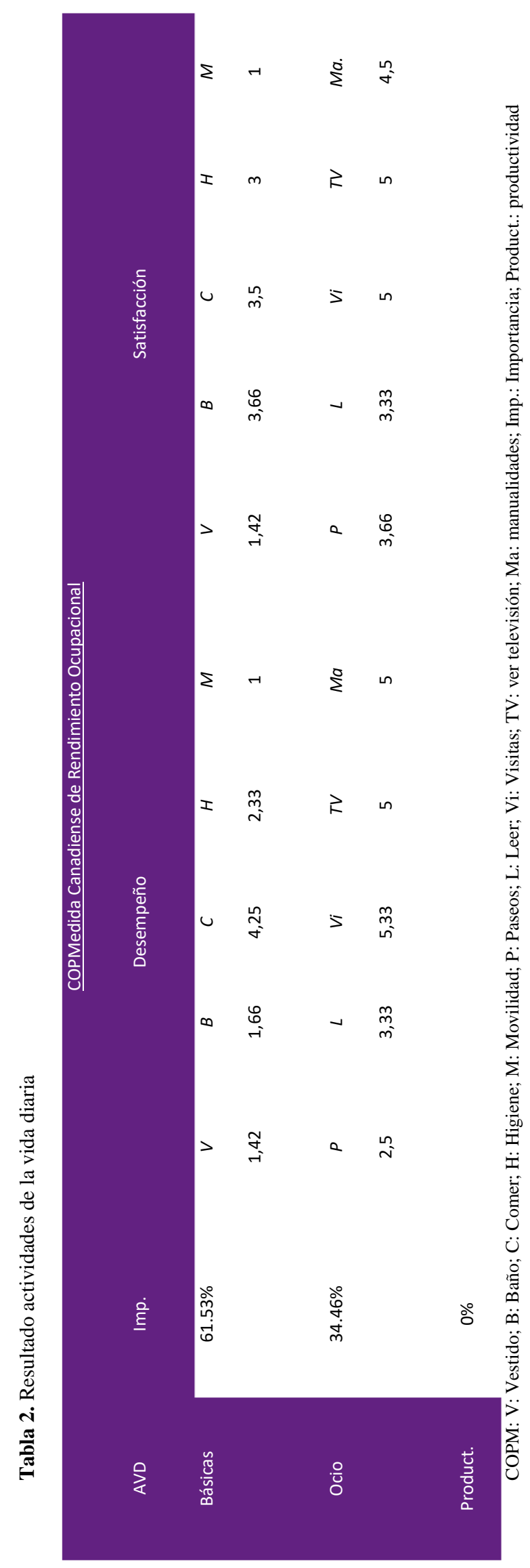


Tabla 3: Resultados actividades de la vida diaria del IB.

una relación

va con el

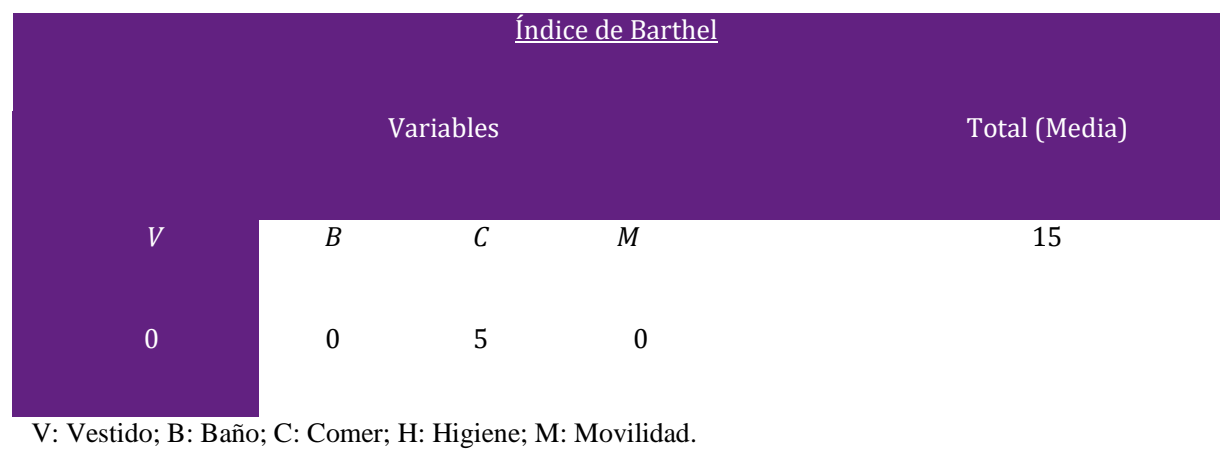

En relación a los datos del desempeño ocupacional obtenidos con la COPM, los pacientes daban mayor importancia las dificultades provocadas en las rutinas relacionadas con las actividades básicas de la vida diaria (ABVD) $(61,53 \%)$ y en menor medida en el ocio $(34,46 \%)$. En relación a las AVD manifiestan tener mayor dificultad en la movilidad, el baño y el vestido siendo las menos afectadas la higiene y la comida.

Estos datos se reproducen en el IB en que se encuentra mayor dependencia en las mismas áreas, puntuación media del IB es 15 puntos. Entre las actividades de ocio que se realizan en el hospital en la que expresan mayor dificultad es en pasear (Tabla 2Tabla 3). A continuación, en las correlaciones de la COPM y el IB con el resto de instrumentos (Tabla 4) se muestran los resultados estadísticamente significativos.

Que la persona sienta que su vida tiene sentido obtiene una relación directamente proporcional y significativa con la satisfacción de cuidado personal de la COPM. Por lo tanto, que el paciente pueda realizar estas actividades de cuidado personal de manera independiente, hace que aumente su grado de satisfacción con respecto a estas actividades, considerando que su vida tiene sentido.

Que la persona sienta energía suficiente para la vida diaria obtiene una relación directamente proporcional y significativa con el desempeño total de la COPM. Cuanta mayor energía siente, mayor desempeño tiene en todas las áreas. Considerar con frecuencia desempeño total de la COPM. Es decir, cuanto mayor es la frecuencia de los sentimientos negativos, menor es el desempeño en todas las áreas.

El dolor nocturno y en bipedestación obtiene una relación inversamente proporcional y significativa con el desempeño en las ABVD de la COPM. Se obtiene resultados en la línea del dominio del dolor del PN en el que las dificultades en estas tareas se ven influenciadas cuando se siente dolor nocturno, en la deambulación y la bipedestación. A su vez, el insomnio obtiene una relación inversamente proporcional y significativa con el desempeño en actividades de cuidado personal. Al disminuir su desempeño, el 58,3\% de los pacientes decían sentirse una "carga".

Que la persona haya olvidado qué es pasarlo bien y que tenga los nervios de punta, obtiene una relación inversamente proporcional y significativa con el desempeño total en actividades de la COPM. Por lo tanto, cuanto mayor es el olvido de pasárselo bien y más nervioso esté, menor es el desempeño en todas las áreas.

La incontinencia y la puntuación total del Norton obtienen una relación directamente proporcional y significativa con los traslados sillón/cama y la puntuación total del Barthel. Cuanto mayor continencia hay y menor riesgo de deterioro de la integridad cutánea y de la presencia de UPP, mayor independencia en los traslados y en general. 


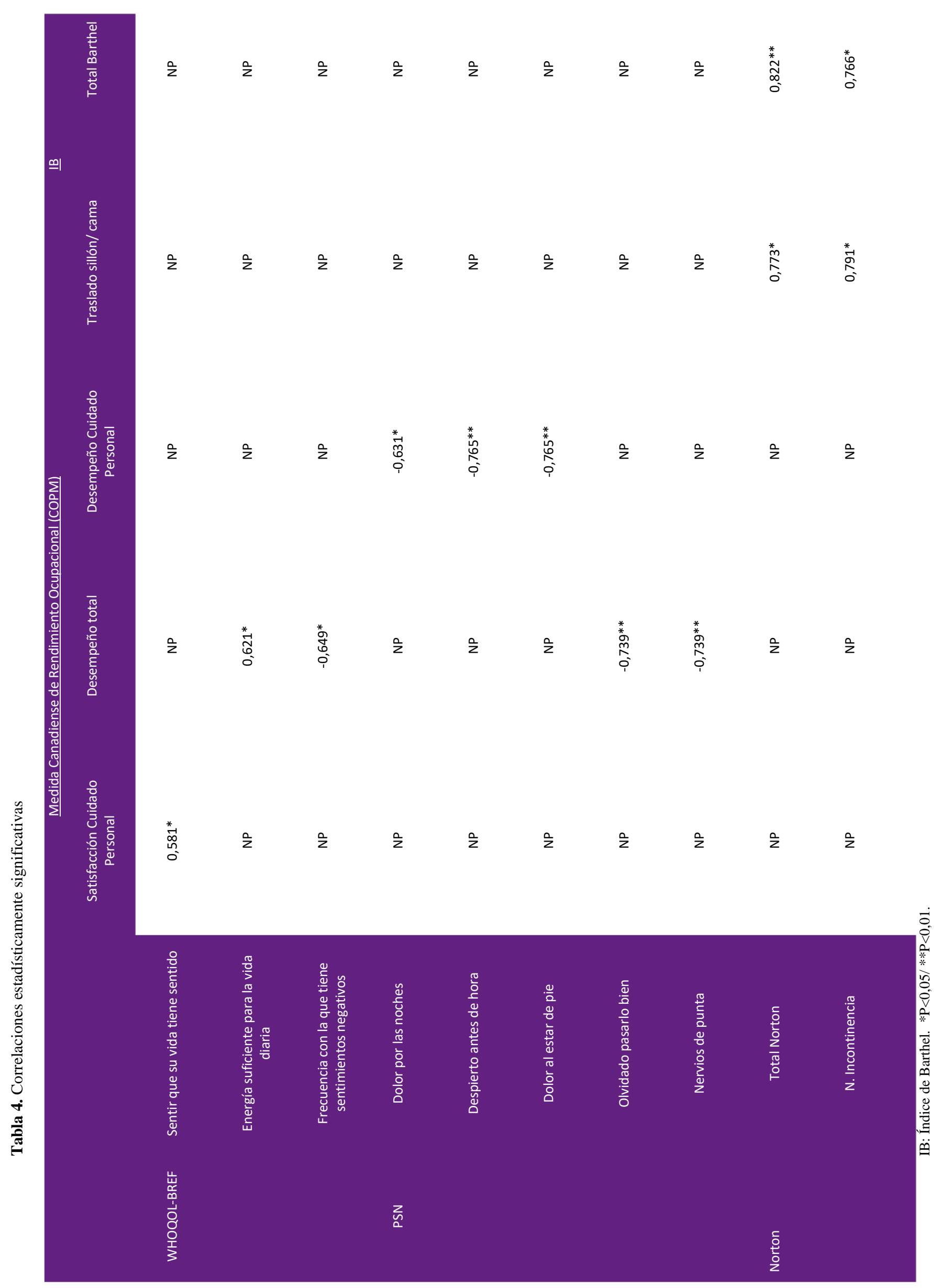


La movilidad obtiene una relación directamente proporcional y significativa con la puntuación total del Barthel. A mayor movilidad tenga, mayor independencia en las AVD.

\section{DISCUSIÓN}

Estos resultados permiten acercarnos al perfil de dependencia de los pacientes con CP para posteriormente plantear una intervención desde la TO con el objetivo de mejorar la $\mathrm{CV}$ de estos pacientes.

Como las características de estos pacientes son muy específicas y muestran gran variabilidad es imprescindible conocer las necesidades y deseos específicos de los mismos para poder establecer un plan de tratamiento eficaz.

El abordaje rehabilitador paliativo desde TO intenta minimizar el impacto de la enfermedad sobre sus capacidades, haciendo especial hincapié en las necesidades concretas del paciente y reduciendo la intensidad de los síntomas de la enfermedad con el fin de promover su independencia y participación en el mayor grado que lo permitan sus circunstancias particulares. Es una parte esencial para potenciar y mantener su autoestima y dignidad y debe apoyarse en la familia (Astudillo Alarcón et al., 2008).

Los estudios que revisan la eficacia de intervenciones no farmacológicas han recibido escasa atención en la literatura, quizá por no existir estudios relacionados con el perfil de los pacientes, generalmente con una baja esperanza de vida que lo dificulta que permita diseñar intervenciones específicas y adaptadas a las necesidades reales de los pacientes.

El control de síntomas clínicos y el mantenimiento de las actividades de autocuidado son los objetivos prioritarios de los pacientes con enfermedad avanzada, lo que está en consonancia con los resultados obtenidos en nuestro estudio (Schleinich et al., 2008). Las actividades de la vida diaria ayudan a crear rutinas diarias básicas que permiten sentirse capaz y útil en cuidado de uno mismo, lo que puede explicar esta prioridad en los pacientes.

Según nuestros resultados de los ítems del dominio del sueño, vemos que el dolor por las noches no influye directamente en su calidad del sueño.

Sin embargo, algunos estudios indican que el insomnio es un factor de riesgo que, junto al resto de los factores específicos del cáncer, aumentan el riesgo de depresión, aunque sus consecuencias son minimizadas frente a ellos (Savard \& Morin, 2001; Irwin, 2013).

La sensación de ser una carga para los cuidadores está muy relacionada con la falta de esperanza e ilusión por la lucha constante por encontrar un sentido en su vida ante la inminencia de la muerte.

Unido a la dependencia de sus cuidadores para las actividades de la vida diaria y las limitaciones en la movilidad, la sensación de ser una carga es frecuente en este tipo de pacientes (Wertheim et al., 2017). Correspondiéndose con los datos de este estudio

La limitación más importante del estudio ha sido el agravamiento sintomático de los pacientes e incluso el deceso, que junto con la fluctuación en los ingresos ha hecho difícil obtener un mayor tamaño de la muestra. No obstante, la muestra es suficiente para una primera impresión sobre la realidad de los pacientes de CP, en relación a la actividad y el desempeño ocupacional.

Para poder definir con mayor exactitud el perfil de los pacientes atendidos en unidades de CP y planificar una intervención adecuada desde TO es imprescindible continuar los estudios en esta área

\section{CONCLUSIÓN}

En este estudio, se ha podido constatar que la disminución del desempeño ocupacional y la dependencia en la realización de actividades de la vida diaria constituyen un problema común en los pacientes de CP. 
Estos presentan un perfil de dependencia severo en la realización de actividades de la vida diaria. Siendo el dolor, el sueño, el estado psicológico, la continencia, las UPP, la movilidad y la energía, factores que limitan su desempeño ocupacional, principalmente en las actividades básicas de la vida diaria.

\section{FRASES DESTACADAS}

$>$ La dependencia en las actividades de la vida diaria es común en pacientes de Cuidados Paliativos.

$>$ La terapia ocupacional facilita la adaptación a la enfermedad del paciente de Cuidados Paliativos

$>$ Realizar actividades significativas para el paciente influye en la mejora de la calidad de vida.

$>$ Los pacientes de Cuidados Paliativos presentan un perfil funcional de dependencia severa.

Una intervención específica de TO dirigida al mantenimiento de las capacidades residuales de los pacientes para la realización de las AVD, el aprendizaje de estrategias y uso de productos de apoyo que le permitan mantener durante más tiempo su independencia en la movilidad, así como la adaptación de las actividades y del entorno, teniendo en cuenta sus síntomas específicos, su gravedad y su influencia tanto física como psicológica y emocional pueden generar mejoras en la percepción de su CV. Las técnicas de TO aplicadas desde un momento temprano de la enfermedad pueden, además, favorecer la adaptación progresiva a la enfermedad y una mejor aceptación del momento de la muerte.

\section{REFERENCIAS}

Alonso J, Prieto L, Antó J. The Spanish version of the Nottingham Health Profile: a review of adaptation and instrument characteristics. Qual LIfe Res. 1994;Dec;3(6):385-93.

Année H. L'ergothérapie et l'activité en soins palliatifs. Rennes: Institut de Formation en Ergothérapie de Rennes FOURNIER; 2012.
Astudillo Alarcón W, Mendinueta Aguirre C, Astudillo-Alarcón E. La rehabilitación y los cuidados paliativos. Med paliativacuidados del enfermo en el Final la vida y atención a su Fam. 2008. p. 591-604.

Barrero Solís C, García Arrioja S, Ojeda Manzano A. Índice de Barthel (IB): Un instrumento esencial para la evaluación funcional y la rehabilitación. Nuevos Horizontes. 2005;4(1-2 Enero-Junio, Julio-Diciembre.).

Corner J. Pain:Clinical manual for nursing practiceMcCaffery,M,Beebe,A. Mosby; 1989.

Corregidor Sánchez A, Ávila Tato R. Terapia Ocupacional en Cuidados Paliativos: ocupar antes de morir. TOG. 2010;185213.

Francis H, Francis L, Downing M, Lespperance M. A reliability and validity study of the Palliative Performance Scale. BMC Palliat Care. 2008;7(10) DOI: http://dx.doi.org/10.1186/1472-684X-7-10.

Gómez-Pavón J, Catalá T. Terapia Ocupacional en la atención al final de la vida. TOG. 2010;185-213.

Instituto de Información Sanitaria. Atención a los Cuidados Paliativos 2010. Organización en las comunidades autónomas.Sistema de información de Atención Primaria (SIAP). Madrid, España: Ministerio de Sanidad, Politica Social e Igualdad; 2010.

Instituto Nacional de Salud. Guía de cuidados de enfermería. Úlceras por Presión. Madrid, España: INSALUD; 1996.

Lindahl-Jacobsen L, Hansen D, Waehrens E, la Cour K, Sonderrgaard J. Performance of activities of daily living among hospitalized cancer patients. Scand J Occup Ther. 2015; Mar, 22(2):137-46.

Mengual F, Pérez J, Sesé G, Pérez M, Cardona R. Paciente terminal. 2005. p. 1-52.

Ministerio de Sanidad Politica Social e Igualdad. Estrategia en Cuidados Paliativos del Sistema Nacional de Salud. Actualización 2010-2014. Madrid, España; 2010.

Irwin MR. Depression and insomnia in cancer: prevalence, risk factors, and effects on cancer outcomes. Curr Psychiatry Rep. 2013;15(11):404.

Savard J., Morin CM. Insomnia in the context of cancer: a review of a neglected problem. J Clin Oncol. 2001;19(3):895-908.

Schleinich M, Warren S, Nekolaichuk C, Kaasa T, Watanabe S. Palliative care rehabilitation survey: a pilot study of patients' priorities for rehabilitation goals. Palliat Med. 2008;22(7):822-3.

Sociedad Española de Cuidados Paliativos. Estatutos de SECPAL. Madrid, España;

Sociedad Española de Cuidados Paliativos. Guía de Cuidados Paliativos. Madrid, España; 2002.

The WHOQOL Grup. Development of the World Health Organization WOQOL-BREF quality of life assessment. Psychol Med. 1998;Mayo; 28(3):551-8.

Wertheim R, Hasson-Ohayon I, Mashiach-Eizenberg M, Pizem N, Shacham-Shmueli E, Goldzweig G. Hide and "sick": Selfconcealment, shame and distress in the setting of psychooncology. Palliat Support Care. 2017;Jun(21):1-9.

WFOT. Definition of Occupational Therapy. 2012. 
